Revue d'histoire de l'Amérique française

RAS REVUE D.HISTOIRE DE L'AMÉRIQUE FRANÇAISE

\title{
Annaotaha et Dollard vus de l'autre côté de la palissade
}

John A. Dickinson

Volume 35, numéro 2, septembre 1981

URI : https://id.erudit.org/iderudit/303948ar

DOI : https://doi.org/10.7202/303948ar

Aller au sommaire du numéro

Éditeur(s)

Institut d'histoire de l'Amérique française

ISSN

0035-2357 (imprimé)

1492-1383 (numérique)

Découvrir la revue

Citer cet article

Dickinson, J. A. (1981). Annaotaha et Dollard vus de l'autre côté de la palissade. Revue d'histoire de l'Amérique française, 35(2), 163-178.

https://doi.org/10.7202/303948ar d'utilisation que vous pouvez consulter en ligne.

https://apropos.erudit.org/fr/usagers/politique-dutilisation/ 


\title{
ANNAOTAHA ET DOLLARD VUS DE L'AUTRE CôTÉ DE LA PALISSADE
}

\author{
JOHN A. DICKINSON \\ Département d'histoire \\ Université de Montréal
}

Les rapports entre Amérindiens et Européens ont depuis longtemps suscité beaucoup d'intérêt parmi les historiens. Cependant, dans leurs interprétations des événements marquants des débuts des colonies américaines, ceux-ci ont le plus souvent tenté de justifier l'action des colons et ont fait peu d'efforts pour comprendre le comportement des Amérindiens. Cet état de choses s'explique en partie par le fait que les sources pour l'étude de cette époque sont essentiellement d'origine européenne et l'appréciation qu'avaient les autochtones des événements est souvent difficile à déterminer. Ainsi l'on aboutit à des interprétations eurocentriques qui embrouillent l'histoire autant qu'elles l'éclairent. Pourtant des indices quant aux motivations des Amérindiens sont assez fréquents dans les textes pour que l'on puisse deviner des explications plausibles de leur comportement et, par ce moyen, atteindre une interprétation plus nuancée des événements.

Les guerres iroquoises illustrent bien ce problème. Depuis quarante ans, les historiens acceptent implicitement ou explicitement la thèse de George $T$. Hunt qui nie toute spécificité du comportement indigène; dès leurs premiers contacts avec des marchands hollandais, les Iroquois auraient adopté une mentalité nettement européenne ${ }^{1}$. Cette version des faits mérite d'être réévaluée car les moeurs d'un peuple ne peuvent être transformées d'une manière aussi complète du jour au lendemain, surtout lorsqu'on a affaire à une culture aussi complexe que celle des Iroquoiens. Chez toutes les tribus amérindiennes, certains traits du comportement ancien persistent bien après les premiers contacts; on n'a qu'à penser à l'importance des songes pour la médecine et la chasse. En ce qui concerne la guerre, la nouvelle technologie a changé les conséquences des hostilités en les rendant plus meurtrières, mais cela n'implique nullement l'abandon des conceptions traditionnelles du

1 George T. Hunt, The Wars of the Iroquois: a Study in Intertribal Relations (Madison, University of Wisconsin Press, 1940), 3-5.

RHAF, vol. 35, no 2, septembre 1981 
comportement attendu d'un guerrier. Le code d'honneur et la nécessité d'apaiser les esprits des leurs, tués par l'ennemi, demeuraient toujours importants pour les guerriers et sont des facteurs qui ne trouvent aucune explication économique. L'analyse d'un fait déjà fertile en controverses, et donc bien connu, servira à illustrer la permanence de certains comportements.

L'exploit du Long-Sault est l'un des événements les plus controversés de l'âge héroïque de la Nouvelle-France. La polémique atteignit son sommet vers 1960 lorsque des articles et des brochures aussi emportés les uns que les autres marquèrent le tricentenaire de cette bataille. Cependant, depuis la parution de l'excellente biographie de Dollard par André Vachon ${ }^{2}$, les historiens ont délaissé le sujet et les feux de la controverse s'éteignent. Il peut paraître inutile de vouloir remuer les cendres à nouveau, mais le débat autour de la personnalité de Dollard a détourné l'attention d'un élément capital de cet événement: la perspective qu'en avaient les Amérindiens. En effet, toute l'historiographie est coupable d'un eurocentrisme très marqué et l'on n'a jamais tenté d'élucider les buts des principaux protagonistes tant Iroquois que Hurons, ni d'étudier leur appréciation de la signification de ce combat.

\section{Les sources}

S'il y a eu tant de controverses, c'est que les sources n'ont pas toujours été évaluées avec autant de rigueur qu'on aurait pu l'espérer. Les seuls témoins oculaires, à part bien sûr les Iroquois, étaient des Hurons qui ont réussi à s'évader et à retourner à Montréal. La principale source est le témoignage du Huron Louis, recueilli par le père Chaumonot à peine trois semaines après la bataille ${ }^{3}$. C'est ce témoignage corroboré par celui de deux ou trois autres rescapés hurons qui constituent la source essentielle de la Relation des Jésuites de $1660^{4}$. André Vachon a établi de façon magistrale la valeur de la source huronne et surtout de l'exposé de Chaumonot ${ }^{5}$. Cependant les partisans de la légende de Dollard ont toujours montré une certaine réticence à l'accepter. Par exemple,

2 André Vachon, "Adam Dollard des Ormeaux", Dictionnaire biographique du Canada (Québec, Les Presses de l'Université Laval, 1966), I: 274-282. Cette biographie extrêmement judicieuse comporte une discussion des principales phases de la controverse et une solide bibliographie. Cependant, et c'est normal, Vachon se préoccupe surtout de la participation française.

3 Cité par Mère Marie de l'Incarnation dans une lettre à son fils du 25 juin 1660 . Marie de l'Incarnation, Lettres (L'abbé Richaudeau, éd.) (Paris, Librairie Internationalecatholique, 1876), II: 154-162.

4 Rueben Gold Thwaites, ed, The Jesuit Relations and Allied Documents (Cleveland, Burrows Bros., 1896-1900), XLV: 240-260, XLVI: 22-62, 108-122 (Ci-après RJ).

5 André Vachon, "La valeur de la source huronne», Revue de l'Université Laval, XVIII (1963): 495-515. 
Lionel Groulx jugeait les Hurons incapables de donner une version correcte de l'événement:

Les récits de Chaumonot et des Relations sont fondés sur des témoignages de Hurons qui appartenaient vraisemblablement au groupe des déserteurs. Ces Hurons n'avaient pas intérêt à se donner le mauvais rôle. Et l'on sait quelles difficultés éprouvaient ces pauvres sauvages à s'exprimer exactement. ${ }^{6}$

Peut-on espérer trouver le moindre effort de compréhension du comportement des Amérindiens après une telle affirmation péremptoire? La seule autre information directe sur le combat provient de la Nouvelle-Hollande et fait état des pertes iroquoises ${ }^{7}$.

Les correspondances de Mère Marie de l'Incarnation ${ }^{8}$ et du gouverneur d'Argenson ${ }^{9}$ sont utiles pour connaître les réactions et l'état d'esprit dans la colonie ainsi que les appréhensions des habitants. Toutefois, les lettres de l'Ursuline sont supérieures à celles du gouverneur qui, comme les Jésuites, avait toujours un souci de propagande. Enfin, le Journal ${ }^{10}$ des Jésuites et l'acte de décès dressé le 8 juin à Montréal ${ }^{11}$ sont peu loquaces sur l'événement. Ces documents constituent les seules véritables sources dont nous disposons.

D'autres écrits ont souvent été utilisés comme sources de première main même s'ils n'ont pas ce caractère. Parmi ceux-ci, le plus problématique est la relation de Pierre-Esprit Radisson. Celui-ci passa devant le petit fort trois mois après l'événement ${ }^{12}$. On peut légitimement douter des conclusions qu'il aurait pu tirer des vestiges du combat; l'information qu'il donne doit nécessairement provenir d'informateurs montréalais ou québécois qui, euxmêmes, n'étaient pas des témoins oculaires. Qui plus est, ce document ne fut rédigé que huit à neuf ans plus tard, et il ne faudrait pas s'attendre à une trop grande précision sauf sur le problème de l'emplacement exact du fort ${ }^{13}$. Rédigées près de 40 ans après l'événement, les quelques lignes consacrées à ce sujet par Marguerite

6 Lionel Groulx, Dollard, est-il un mythe? (Montréal, Fides, 1960), 15.

7 Lettre de La Montagne, facteur à Fort Orange, cité par Jacques Rousseau, «L'affaire Dollard, de Fort Orange au Long-Sault", Revue d'histoire de l'Amérique française, XIV, 3 (Décembre 1960): 370-377.

8 Marie de l'Incarnation, op. cit., II: 148-166, 187-191.

9 Cité par Adrien Pouliot et Silvio Dumas, L'exploit du Long-Sault. Les témoignages des contemporains (Québec, Société Historique de Québec, 1960), «Cahier d'histoire no $12 »: 46-49$.

10 Le Journal des Jésuites (Laverdière et Casgrain, éds.) (Montréal, Éditions François-Xavier, 1973), 284 (ci-après JJ).

11 Reproduit dans Édouard-Zotique Massicotte, Dollard des Ormeaux et ses compagnons (Montréal, Le comité du monument Dollard des Ormeaux, 1920), 70-71.

12 Cité par Pouliot et Dumas, op. cit.: 50-53.

13 Grace Lee Nute, «Pierre-Esprit Radisson», Dictionnaire biographique du Canada (Québec, Les Presses de l’Université Laval, 1969), II: 558-560. 
Bourgeoys, qui était alors âgée de 78 ans, ne constituent pas une information très sûre ${ }^{14}$. De plus, sa mémoire confond deux épisodes bien distincts. Enfin il y a l'Histoire du Montréal du sulpicien François Dollier de Casson. Son récit est l'un des plus complets et il constitue l'origine de la légende Dollard ${ }^{15}$. Cependant, l'auteur n'est arrivé au Canada qu'en 1666 et il n'a rédigé son histoire qu'une dizaine d'années après le combat. Il n'a pu consulter aucun témoin oculaire de l'événement et il a dû se fier aux souvenirs des anciens Montréalais ${ }^{16}$. Ainsi, il est impossible de déterminer quelle part de son récit est fondée sur des faits, quelle part sur la spéculation et quelle part tient de l'invention pure et simple. Il est certain que Dollier manquait d'objectivité. Il tente toujours de donner le beau rôle aux Montréalistes en soulignant leurs actions édifiantes ou héroïques. Par ailleurs, il n'a aucune sympathie pour les Amérindiens. Les Hurons ne sont pas des personnages importants dans son Histoire et, lorsqu'il en parle, c'est pour les dénigrer. Il les qualifie de "lâches»" ${ }^{17}$, de "marchandise mêlée» ${ }^{18}$ et de "paignots» ${ }^{19}$; il affirme qu'ils ont une «inclination fort portée à la trahison ${ }^{20}$. Malgré ces défauts, Dollier a souvent été considéré comme la meilleure «source» et précisément parce qu'il n'avait aucun attachement aux «sauvages». C'est notamment le point de vue de Faillon et de Groulx ${ }^{21}$. Pour essayer de comprendre le comportement des Amérindiens, Dollier de Casson est peu utile.

\section{Le comportement huron}

La Relation et le Journal des Jésuites donnent la priorité aux guerriers hurons dans leur compte rendu de l'exploit du LongSault ${ }^{22}$. Cela est normal puisque les Jésuites avaient la charge des Hurons mais non pas de Villemarie. Ces guerriers étaient partis de Québec à la fin de l'hiver pour faire la «petite guerre» aux Iroquois comme ils le faisaient chaque année ${ }^{23}$. Les objectifs de ces expéditions étaient doubles: tuer quelques ennemis pour venger la mort

14 Cité par l'abbé Etienne-Michel Faillon, Histoire de la colonie française au Canada (Villemaire, Bibliothèque paroissiale, 1865-66), II: 414.

15 François Dollier de Casson, Histoire du Montréal (Flenley ed.) (London \& Toronto, Dent, 1928), 252-266.

16 Il reconnaît que son histoire peut comporter des erreurs et aussi que la mémoire n'est pas toujours fidèle: «Je me contente seulement de vous rapporter les plus notables actions qui se firent pour lors, les autres étant hors le souvenir des hommes.» Ibid, 154.

17 Ibid., 106, 285.

18 Ibid., 108.

19 Ibid., 258.

20 Ibid., 256.

21 Faillon, op. cit., II: 409-410; Groulx, op. cit., 15

22 Le chapitre pertinent dans la Relation s'intitule: «De l'Estat du reste des Hurons et de sa dernière défaite par les Iroquois», RJ, XLV: 240-260. Dans le Journal on lit: «le 8 sur la minuit vint la nouvelle de la défaite des 40 Hurons...», JJ: 284.

23 Malgré la paix entre Iroquois et Français, les Hurons et Algonkins continuaient à attaquer. Par exemple, JJ:225 (1657); 234, 236 (1658); 263-264 (1659). 
des leurs et prendre des prisonniers pour servir de monnaie d'échange contre les Hurons et les Algonkins captifs en Iroquoisie. D'après le récit de Chaumonot, l'expédition de 1660 avait un autre but. Les Hurons, déjà fort décimés par les enlèvements répétés des leurs, espéraient faire subir une assez grande défaite aux Iroquois pour les empêcher de venir à nouveau les menacer à Québec. Les paroles attribuées à Eustache Thaouonhohoui sont très précises sur ce point:

Vous savez, ô bienheureux habitants du ciel, ce qui nous a conduits ici, vous savez que c'est le désir de réprimer la fureur de l'Iroquois, afin de l'empêcher d'enlever le reste de nos femmes et de nos enfants, de crainte qu'en les enlevant ils ne leur fassent perdre la Foi, et ensuite le paradis les emmenant captifs en leur pays. ${ }^{24}$

Sans doute ne s'attendaient-ils pas à rencontrer une troupe aussi nombreuse, mais plutôt des petites bandes de 10 à 20 guerriers. C'est ce qui explique qu'ils se soient dirigés vers l'Outaouais plutôt que vers le Richelieu.

Les Hurons accompagnés de quatre Algonkins qui s'étaient joints à eux à Trois-Rivières ont rencontré Dollard et ses seize compagnons près de Montréal ${ }^{25}$. Si Dollard était incontestablement le chef des Français et Étienne Annaotaha le capitaine des siens, le commandement suprême des alliés pose des problèmes. Quelques indications suggèrent qu'Annaotaha était le plus influent. Lors de la première rencontre avec les avant-coureurs iroquois, un Huron "opina qu'il fallait descendre à Montréal» puisque le succès de la mission était désormais compromis. Annaotaha résista et «on suivit le sentiment de ce dernier» ${ }^{26}$. Ce fut encore le chef huron qui proposa l'envoi d'une ambassade pour essayer de trouver un compromis après l'arrivée des Agniers et Onneyouts. Encore une fois «son sentiment fut suivi» ${ }^{27}$. La seule fois que les Français prirent une décision de leur propre chef, ce fut pour faire feu sur les Iroquois alors que les pourparlers étaient encore en cours, et ce geste précipita le massacre de ceux qui restaient dans le

24 Marie de l'Incarnation, op. cit., II: 158.

25 La version de Dollier de Casson fait arriver les Hurons plusieurs jours après le départ de Dollard. Ceci est possible, bien que les Jésuites affirment que tous sont partis ensemble de Montréal. Beaucoup moins probable est l'histoire de la lettre envoyée par de Maisonneuve à Dollard lui disant de se méfier des Hurons. Dollier considérait les Hurons responsables de ce désastre et en attribuant cette "perspicacité» à de Maisonneuve il ne faisait qu'agrandir la renommée de son héros principal. De toute façon, on peut légitimement se demander d'où Dollier aurait eu cette information car il n'a jamais pu discuter de cette affaire avec le fondateur de Montréal qui avait quitté la colonie avant l'arrivée du sulpicien.

26 Marie de l'Incarnation, op. cit., II: 154-155.

27 Ibid., II: 158. 
fort $^{28}$. Cette hypothèse d'un commandement autochtone n'a pas eu la faveur des historiens qui préfèrent Dollard (est-ce par eurocentrisme?), mais elle ne doit pas être écartée trop hâtivement. Annaotaha était un chef assez âgé, déjà bien aguerri, et qui comprenait parfaitement toutes les ruses des Iroquois. Dollard était un jeune homme qui avait à peine atteint la majorité. Dans toutes les sociétés à cette époque, l'âge et l'expérience méritaient certains égards. De plus, ça ne faisait qu'une année que Dollard était au pays: son manque d'expérience dans les méthodes de la petite guerre ainsi que son ignorance apparente de la diplomatie amérindienne le désavantageaient. Quoi qu'il en soit, une chose est certaine: un groupe d'hommes d'âge mûr ne se laisseraient pas mener par un jeune sans expérience.

Pendant le siège du petit fort, les Hurons se sont battus vaillamment durant sept jours. Cependant, leur habitude de répondre à chaque coup de feu de l'ennemi rendit la situation des défenseurs assez précaire. Leurs munitions étaient épuisées avant l'arrivée des Agniers et le plomb commençait à manquer aux Français ${ }^{29}$. Devant cet état de choses, à l'arrivée d'un renfort de 500 hommes pour l'ennemi, Annaotaha tenta le seul expédient possible: une ambassade. C'est sous la couverture de cette trêve que des Hurons iroquoisés sont venus solliciter leurs parents de se joindre à eux pour sauver leurs vies. Vingt-quatre Hurons se sont "envolés»; cette action attira l'opprobre des historiens, mais elle était très rationnelle de leur point de vue. Sans munitions, la défense était désormais impossible. Si l'ambassade réussissait, ils savaient bien que le mieux qu'ils pouvaient espérer était de devenir des prisonniers de guerre. Se joindre volontairement aux Iroquois leur permettrait peut-être de vivre, résister encore entraînerait leur mort. Les Français ne pouvaient comprendre ce qui se passait et ils brisèrent la trêve en tirant sur les Iroquois qui s'étaient le plus rapprochés du fort. Ce geste, considéré comme déloyal par les Iroquois, scella le sort des défenseurs; et ainsi Dollard et ses compagnons ont précipité leur propre mort. Annaotaha l'avait bien compris et il leur en fit le reproche:

Ah! camarades vous avez tout gâté, encore deviez vous attendre le résultat du conseil de nos ennemis. Que savons-nous s'ils ne demandent point à composer, et s'ils ne nous accordent point de nous séparer les uns des autres sans acte d'hostilité, comme il est souvent arrivé en de semblables rencontres? ${ }^{30}$

28 Ibid., II: 159-160.

29 Ibid., II: 157.

30 Ibid., II: 159-160. 
En effet, il est fort possible que l'ambassade aurait abouti à un compromis par lequel les Hurons et les Algonkins auraient fait les frais de la rencontre, tandis que les Français auraient eu la vie sauve. Cela dépend bien sûr du véritable motif des Iroquois, qu'une analyse de leur comportement tentera d'établir.

\section{Le comportement iroquois}

D'après les historiens, les Iroquois voulaient détruire la Nouvelle-France et chasser tous les Blancs de l'Amérique du Nord à l'exception des Hollandais ${ }^{31}$. Mais était-ce vraiment le cas? Cette version permet une reconstitution dramatique du passé québécois, mais ne colle pas toujours à la réalité. Pour les événements de 1660 , une source semble confirmer cette interprétation: les révélations d'un captif iroquois qui affirme sous torture que deux armées composées de 900 à 1200 hommes au total devaient se joindre «pour venir ensuite tous ensemble fondre sur Québec. Il ajoutait que leur dessein était d'enlever la tête d'Ononthio, qui est M. le Gouverneur, afin que le chef étant mort, ils puissent plus facilement mettre tout le pays à feu et à sang» ${ }^{32}$. Que vaut un aveu extorqué sous torture? Par ailleurs, les guerriers iroquois se devaient d'être courageux devant ce supplice et d'exagérer leurs prouesses et leur valeur. Or nous savons que l'armée n'était composée que d'environ 700 hommes ${ }^{33}$. Aussi serait-il légitime pour le détenu d'en vouloir au gouverneur de l'avoir destiné au bûcher; dans les années précédentes, les prisonniers étaient mis aux fers, mais ils ne subissaient pas la torture. Son honneur réclamait que ses compatriotes vengeassent sa mort en scalpant le gouverneur. Ce seul témoignage, qui épouvanta les habitants de Québec et notamment $\mathrm{Mgr}$ de $\mathrm{Laval}^{34}$, est à l'origine de la légende voulant que Dollard aît «sauvé» la colonie de la menace iroquoise. Cependant, pour comprendre le comportement des Iroquois, il faut remonter de quelques années, jusqu'au début de la paix de 1653.

La paix fragile inaugurée à l'automne de 1653 ne fut jamais comprise par les Français ${ }^{35}$. Ceux-ci voulaient une paix universelle qui garantirait l'approvisionnement de la colonie en fourrures et

${ }^{31}$ Groulx, op. cit., 41. C'est un exemple parmi d'autres où mène cet argument. Parmi les rares historiens à nier ces buts mentionnons: E.R. Adair, «Dollard des Ormeaux and the fight at the Long-Sault. A reinterpretation", Canadian Historical Review,, XIII (1932): 136; Léo-Paul Desrosiers, "Il y a trois cents ans», Cahiers des Dix, 25 (1960): 93.

32 Marie de l'Incarnation, op. cit., II: 149.

33 Dans les rencontres, l'exagération du nombre était assez fréquente. Lorsque Annaotaha et sa bande rencontrent les Onontagués, ils prétendent être 100 guerriers et pourtant ils ne sont que 61. Ibid., II: 155 .

34 C'est à la suite de cet aveu que l'évêque ordonna aux Ursulines de quitter leur couvent pour se réfugier chez les Jésuites. Marie de l'Incarnation jugea cette action exagérée puisqu'elle se sentait en sécurité au couvent. Ibid., II: 149 et JJ: 282.

35 RJ. XLI: 42-64. 
qui écarterait tout risque pour les habitants et les nations alliées. Il est clair que les Iroquois ne partageaient pas cette conception du traité qu'ils venaient de conclure. Ils cherchaient la neutralité des Européens, ce qui leur permettrait de poursuivre leur guerre traditionnelle avec les Hurons, les Algonkins et les autres tribus plus éloignées. Les tenants de la thèse de Hunt cherchent toujours l'explication économique compréhensible pour l'homme moderne, et ainsi toute expédition a un but commercial. Cette explication est séduisante dans sa simplicité, mais la réalité est beaucoup plus complexe. Par exemple, en 1656, les pères Chaumonot et Dablon en route pour Onontagué rencontrèrent un groupe d'Onneyouts et tentèrent de les dissuader d'attaquer les Outaouais qu'accompagnait Chouart des Groseillers. Ils durent renoncer à cette tentative lorsqu'ils apprirent: «qu'ils étaient vivement piqués de la mort de quelques-uns des leurs, qu'ils voulaient venger, à quelque prix que ce fut.» ${ }^{36}$ En 1658, une autre armée partit contre les Outaouais pour venger la mort de 30 Agniers tués en $1657^{37}$. À l'époque, les Outaouais étaient les principaux partenaires commerciaux des Français, et il est certain que les colons européens ressentaient surtout sur leur commerce les effets qu'entraînaient ces attaques. Mais faut-il toujours attribuer un raisonnement européen aux Iroquois? L'esprit de vengeance n'explique-t-il pas mieux cet acharnement à persévérer dans des guerres qui détruisaient tous les partenaires potentiels des Iroquois?

L'hostilité envers les Français est loin d'être établie d'une manière certaine. Dès 1654, Marie de l'Incarnation rapporte que les Agniers avaient dit "qu'ils ne voulaient point de mal aux Français, mais aux Hurons et Algonkins, et qu'ils les voulaient tous tués» ${ }^{38}$. En 1655, les Agniers confirment la paix avec les Français et ajoutent «qu'ils continueraient la guerre contre les Algonkins et contre les Hurons; et qu'ils en massacreraient autant qu'ils en pourraient rencontrer au dessus de la Bourgade Française, nommée des Trois-Rivières ${ }^{39}$. Encore en 1658, le chef Garakontié, véritable ami des Français, demande au gouverneur par des présents: «23e Laisse moi passer quand je viendrai tuer l'Algonkin. $24 \mathrm{e}$ Cherchons un expédient pour que je tue l'Algonkin.» ${ }^{40}$ L'acharnement des Iroquois contre les Algonkins et surtout contre les Hurons ne peut s'expliquer par une motivation purement éco-

36 RJ, XLII: 76.

37 RJ, XLIV: 204. Le père Le Moyne qui est bien au courant de la situation puisqu'il demeure à Agnier écrit que le dessein des Iroquois est d'exterminer tous les Algonkins et Hurons. RJ, XLIV: 218.

38 Marie de l'Incarnation, op. cit., II: 73.

39 RJ, XLI: 216

40 JJ: 242. 
nomique, ni même militaire. En 1653, il ne restait qu'entre 500 et 600 Hurons chrétiens sur l'île d'Orléans, et ce «débris» ne représentait aucune menace sérieuse pour la puissante confédération iroquoise $^{41}$.

L'éminent ethnohistorien, Bruce Trigger, fournit une explication du comportement des Agniers. Il affirme:

The majority of Huron were killed or captured as a result of the general warfare that was going on between the Mohawk and the French; however, the emphasis that the Mohawk placed on capturing Huron prisoners reflected their long-term ambition to incorporate all of the Huron who had come to Quebec into their own society or, failing this, to kill them. ${ }^{42}$

Ainsi les Français seraient privés d'alliés connaissant les méthodes de la guérilla et les Agniers pourraient accroître le nombre de leurs guerriers. Cependant, la première proposition provient d'une acceptation de la thèse de Hunt, et l'inverse de cette proposition est également plausible. Ce qui signifie que la majorité des Français qui furent tués ou capturés le furent à cause de la guerre entre Hurons et Agniers. D'ailleurs, Marie de l'Incarnation semble être de cet avis:

Ces nations se haïssent au dernier point, à cause des massacres qu'ils ont fait les uns sur les autres. C'est là la cause du mal que souffrent nos Français, car comme ils sont obligés de soutenir nos nouveaux chrétiens, ils sont souvent enveloppés dans leurs querelles et dans leurs différends. ${ }^{43}$

C'est le désir d'enlever les Hurons qui est capital et qui explique le comportement des Iroquois entre 1653 et 1660. Les Hurons devaient périr. Leur crime était d'avoir refusé de se joindre aux tribus qui avaient dévasté le pays des Hurons entre 1648 et 1650 . Ce refus était un affront à l'honneur des vainqueurs et exigeait une punition exemplaire. La permanence dans la psyché amérindienne d'un esprit de vengeance complètement incompréhensible pour les Européens est la principale raison de cet acharnement meurtrier.

Pendant la période 1653 à 1660, les Français ne furent jamais directement visés par les diverses bandes iroquoises dans la vallée du Saint-Laurent car les Iroquois n'étaient pas assez unifiés pour affronter ouvertement la Nouvelle-France. Dès le mois de juillet 1654, les Agniers commençaient à être jaloux des tribus supérieu-

41 RJ, XLI: $136-138$.

42 Bruce G. Trigger, The Children of Aataentsic. A History of the Huron People to 1660 (Montréal, McGill - Queen's University Press, 1967), II: 805.

43 Marie de l'Incarnation, op. cit., II: 88. 
res et firent part de leur mécontentement aux autorités de la colonie:

N'est-ce pas par la porte qu'il faut entrer en la maison et non par la cheminée, et par le toit de la cabane, sinon qu'on soit voleur, et qu'on veuille surprendre le monde? Nous ne faisons qu'une cabane, nous autres cinq Nations Iroquoises; nous ne faisons qu'un feu, et nous avons de tout temps habité sous un même toit. (...) Quoy donc vous n'entrez pas dans la cabane, par la porte, qui est au bas estage de la maison? C'est par nous autres Annierhonnons qu'il fallait commencer. Vous voulez entrer par le toit et par la cheminée, commençant par l'Onnontaehronnon. N'avez-vous point de crainte que la fumée ne vous aveugle, nostre feu n'estant pas esteint? ne craignez-vous point de tomber du haut en bas, n'aiant rien de solide où poser vos démarches? ${ }^{44}$

Les Agniers voulaient contrôler tous les échanges entre les Européens et les tribus situées à l'ouest, mais cette ambition menaçait l'existence même de la confédération iroquoise. À cette époque, le père Le Moyne espérait que les Iroquois supérieurs s'uniraient contre les Agniers ${ }^{45}$. Les Jésuites avaient une bonne appréciation des avantages commerciaux que retireraient les Onontagués et les Tsonnontouans d'une alliance avec les Français, mais ils savaient aussi que les Agniers tenteraient de maintenir leur position de force. C'est cette situation qui amena des guerriers Agniers à massacrer une ambassade de Tsonnontouans en $1657^{46}$. En 1660, une ambassade de Goyogouins affirma que les leurs n'avaient jamais attaqué les Français, et l'information que nous avons quant à l'identité des bandes qui rôdaient dans le Saint-Laurent confirme que ni les Goyogouins ni les Tsonnontouans n'aient été hostiles pendant cette période ${ }^{47}$. C'étaient surtout les Agniers et les Onneyouts qui faisaient des ravages. Cependant, les Jésuites et le gouverneur se méfiaient de ces affirmations, et les Français traitaient toutes les tribus sur le même pied.

Certes il y a eu des dégâts, mais même ces incidents ne révèlent aucun dessein bien arrêté de détruire la colonie française car, à presque chaque occasion, les Iroquois s'empressèrent de rétablir les bonnes relations. En 1655, le frère Jean Liégeois qui faisait la sentinelle pour un groupe de travailleurs hurons et algonkins fut

\footnotetext{
44 RJ, XLI: 86-88. Les Agniers font allusion ici à l'envoi d'une mission chez les Onontagués.

45 RJ, XLI: 200-202; Marie de l'Incarnation, op. cit., II: 84.

46 RJ, XLIII: $98-102$.

47 RJ, XLVI: 116-118. Le seul historien qui insiste sur les divisions entre les nations pacifiques (Tsonnontouans, Goyogouins et une partie des Onontagués) et les nations décidément hostiles (Onneyouts et Agniers) est Léo-Paul Desrosiers, loc. cit.: 93 et «Les années terribles», Cahiers des Dix, 26 (1961):68.
} 
tué $^{48}$. À la même époque, le couple Moyen fut abattu sur l'île aux Oies, et leurs enfants enlevés. Quelques jours plus tard, quatre serviteurs du sieur Denis furent attaqués. Mais voilà qu'une délégation ramena les prisonniers et s'excusa de ces incidents qu'elle attribua à une jeunesse indisciplinée ${ }^{49}$. L'explication de la délégation est vraisemblable. Les jeunes guerriers se devaient de réussir un bel exploit. S'ils ne trouvaient pas leur proie favorite (algonkine ou huronne), un colon risquait de devenir la victime car il eût été déshonorant de rentrer bredouille au village. En 1656, ce fut le père Léonard Gareau qui fut blessé à mort entre Trois-Rivières et Montréal. Il accompagnait une troupe de Hurons qui partait pour l'ouest. Les assaillants déposèrent le père à Montréal: «Jettant deux méchans petits présens, selon leur coustume: l'un pour témoigner qu'ils estoient marris de l'accident qui estoit arrivé; l'autre pour essuyer nos larmes et appaiser nos regrets. ${ }^{50}$ Sans doute les deux «méchans petits présens» n'étaient pas considérés comme une réparation digne de ce bon père par les Jésuites, mais pour les Iroquois l'affaire était réglée d'une manière satisfaisante, selon leurs usages. S'ils avaient vraiment voulu faire du mal aux Français, ils n'auraient jamais ramené le père vivant à Montréal et n'auraient éprouvé aucune obligation d'offrir des présents pour apaiser «nos» regrets.

À plusieurs reprises, les Français furent prévenus du danger qu'il y avait à attirer les Hurons près de leurs habitations. En 1657, lorsque les Agniers vinrent chercher les Hurons de l'île d'Orléans, le chef de la mission dit au gouverneur: "Onontio, ouvre tes bras et laisse aller tes enfans de ton sein, si tu les tiens plus longtemps si serrez, il est à craindre qu'on ne te blesse, quand nous les voudrons frapper lors qu'ils l'auront mérité. ${ }^{51}$ Peut-on espérer un exposé plus clair de l'objectif des Agniers? Cependant tous les Hurons ne partirent pas, et il en résulta d'autres incidents. En octobre 1657, trois hommes furent tués à Montréal (Nicolas Godet, Jean SaintPère et Jacques Noël) par la jeunesse onneyoute. Quatre jours plus tard, une délégation arriva avec sept présents pour «essuyer le sang» et "calmer les esprits» ${ }^{52}$. On rapporte, par ailleurs, que les meurtriers auraient dit: «Les François tiennent entre leurs bras les Hurons et les Algonkins, il ne faut donc pas s'étonner, si en vou-

48 François Du Creux, The History of Canada or New France (Robinson et Conacher eds.) (Toronto, Champlain Society, 1952), II: 689-690. Il est à remarquer que si Liégeois n'avait pas été avec les Algonkins sa vie n'aurait pas été en danger car le but de la bande était «to massacre the Algonquins and the Montagnais».

49 Marie de l'Incarnation, op. cit., II: 86-88.

50 RJ, XLII: 236.

51 RJ, XLIII: 188.

52 RJ, XLIV: 194. 
lant frapper les uns, les coups tombent quelquefois sur les autres. $\|{ }^{33}$ Cependant, les Français refusèrent d'accepter l'accommodement proposé par les Iroquois et ils commencèrent à mettre tous les hommes qui visitaient les habitations françaises aux fers, suivant les ordres du gouverneur ${ }^{54}$. Malgré les mauvais traitements qu'ils avaient reçus, les Onneyouts recherchaient toujours la paix, et une délégation revint en 1659 avec cinq présents «pour étouffer tous les sentiments de vengeance $\rangle^{55}$. Les Iroquois avaient fait tout ce que leurs moeurs requéraient pour empêcher cette affaire de dégénérer en guerre ouverte. D'ailleurs, les Français avaient été bien avertis que leur conduite pourrait mener à des hostilités ${ }^{56}$. L'emprisonnement et la contrainte par les fers étaient considérés comme indignes d'un guerrier iroquois, surtout lorsque celui-ci estimait qu'il avait été capturé d'une manière déloyale. Il n'est pas surprenant que la patience des Iroquois devant ce traitement fût rendue à bout en 1660. D'ailleurs, le seul reproche que l'on fit aux captifs français après la bataille du Long-Sault fut: «il m'a mis les fers aux pieds. ${ }^{57}$

L'épisode le plus curieux de cette longue trève est la fuite, à la fin de mars 1658, de 53 Français qui avaient établi une mission chez les Onontagués à Sainte-Marie de Gannentaha ${ }^{58}$. D'après les Jésuites, les Iroquois voulaient massacrer toute la mission dès que les captifs iroquois encore détenus seraient libres. Marie de l'Incarnation écrivit à son fils: "La résolution était, après avoir défait les Français qui étaient sur le lieu, de venir ici sous une amitié déguisée, pour mettre tout à feu et à sang. $/{ }^{59}$ Cette version des faits a été acceptée par tous les historiens, mais elle n'est pas la seule explication plausible. Les Onontagués, chez qui cette mission était établie, semblent avoir été assez divisés quant à l'attitude à adopter vis-à-vis des Français. Cette division rappelle les luttes entre chrétiens et traditionalistes qui déchirèrent la confédération huronne pendant les années $1640^{60}$. Il est difficile d'évaluer avec précision la

53 RJ, XLIV: 216.

54 RJ, XLIV: 154-156, 192-200; JJ: 221-225. D’après Adrien Pouliot le meurtre de Godet est «le tison de plus en plus ardent de la discorde qui déclencherait finalement les événements de 1660». "La menace iroquoise de 1657 à 1660», Revue de l'Université Laval, XV (1960-61): 431. Si tison il y a, c'est plutôt la prise déloyale des Iroquois et leur mise aux fers.

$55 \mathrm{JJ}: 251$.

56 En avril 1658 le père Le Moyne reçut les présents suivants des Agniers: «5. Qu'on laisse aller nos neveux. 6. Tu ne me mettras pas aux fers. 7. Fais comme le Hollandais qui ne se mêle pas des guerres des Loups etc. 8. Vous autres Hollandais, soyez témoins que je dis au Français qu'il commence le premier». JJ: 235.

57 Marie de l'Incarnation, op. cit., II: 161.

58 RJ, XLIV: 148-220.

59 Marie de l'Incarnation, op. cit., II: 129.

60 Trigger, op. cit., II: 710-724, 744-750. 
force des factions opposées à Onontagué, mais il semble qu'une majorité craignait l'influence des prêtres tandis qu'une minorité, sous Garakontié, recherchait sincèrement la paix. Dès l'arrivée des Jésuites, des Hurons iroquoisés et traditionalistes avaient tenté de les faire expulser. Le père René Ménard rapporta que des rumeurs circulaient parmi les Goyogouins dès 1656 à l'effet que les prêtres semaient la mort et la désolation sur leur passage ${ }^{61}$. On se rappellera aussi que le père Isaac Jogues fut tué parce que la maladie s'était déclarée dans le village où il avait laissé une petite cassette noire. Les Iroquois considéraient que cette boîte causait la maladie ${ }^{62}$. Même chez une tribu alliée comme les Hurons, il y avait eu de nombreuses tentatives de renvoyer ou d'assassiner les missionnaires ${ }^{63}$. Les Jésuites étaient sans doute convaincus qu'il y avait un grand danger, et le récit d'une fuite quasi miraculeuse était plus susceptible d'intéresser et d'édifier leurs lecteurs français. Dans cette affaire, le motif des Onontagués n'est pas très clair, et il semble un peu exagéré de conclure qu'ils voulaient massacrer tous les Français au Canada.

La fuite de la mission risquait d'aigrir davantage les relations entre Iroquois et Français. Les Onontagués, et notamment Garakontié, reprochèrent cette fuite aux Jésuites et les invitèrent à retourner à Gannentaha dès septembre $1658^{64}$. Une autre délégation, des Onneyouts cette fois, réclama le retour des prêtres en avril $1659^{65}$. Un dessein de guerre générale contre les Français n'est pas évident dans le comportement des Iroquois. En novembre 1658, huit hommes furent pris à Trois-Rivières, mais libérés avant quinze jours par les Agniers ${ }^{66}$. Ceux-ci cherchaient toujours à libérer les leurs, et leur but en prenant des captifs semble avoir été de montrer qu'ils pouvaient faire du mal aux Français s'ils le voulaient, mais que cela n'était pas leur dessein. Même en 1660-61, au plus fort des hostilités, bon nombre de Français sont tués en attaquant des bandes ennemies. Ces actions offensives entraînaient d'autres pertes puisque les Iroquois devaient venger leurs guerriers. Si les Iroquois avaient vraiment voulu massacrer tous les colons, comment expliquer que presque tous les captifs aient été libérés et que, pendant leur séjour en Iroquoisie, ils n'avaient pas

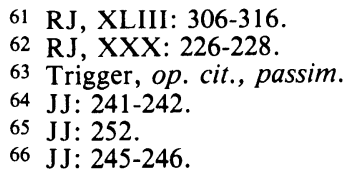


été mutilés mais traités comme des membres de la famille ${ }^{67}$. Le comportement des Iroquois vis-à-vis des Français est, somme toute, assez contradictoire et difficile à déchiffrer. Il semble bien que les Tsonnontouans et les Goyogouins soient demeurés neutres. Les Onontagués étaient divisés, et seuls les Agniers et Onneyouts étaient plutôt hostiles. Cependant cette hostilité n'allait pas jusqu'à vouloir exterminer tous les Français. Ils vengeaient la mort ou le mauvais traitement des leurs ${ }^{68}$ suivant les préceptes de leur code moral.

Le comportement vis-à-vis des Hurons ne souffre d'aucune contradiction: il fallait que cette nation disparaisse complètement, soit par la mort, soit par l'incorporation aux tribus iroquoises. En 1659, Marie de l'Incarnation rapporte des nouvelles d'une armée qui venait pour «enlever nos nouveaux chrétiens, et comme je crois, autant de Français qu'ils pourraient ${ }^{69}$. La première partie de cette affirmation est une certitude, la seconde est une opinion, et il faut bien faire la différence. Depuis 1653, les Iroquois avaient un but principal en venant au Canada: l'enlèvement des restes de la nation huronne. Le 18 novembre 1653, les Jésuites apprirent que les ambassadeurs Agniers avaient présenté secrètement trois présents «d'une rare beauté» aux Hurons pour les attirer dans leur pays $^{70}$. Au mois de février 1654, c'est au tour des Onontagués d'inviter les Hurons à se joindre à eux en présentant quatre présents «dans l'obscurité et dans l'horreur de la nuit»" ${ }^{71}$. Les Hurons hésitaient, craignant d'être victimes d'une supercherie et ils réussirent à temporiser. Cependant, les Agniers et les Onontagués, principaux intéressés, s'impatientaient et, en mai 1656, une bande d'Agniers attaqua la mission huronne sur l'île d'Orléans, à la sortie de la messe. Près d'une centaine de Hurons furent tués ou enlevés ${ }^{72}$. Il est à remarquer que, lors de cet événement, les Fran-

${ }^{67}$ Marie de l'Incarnation, op. cit., II: 213; RJ, XLVII: 200-218. Certains qui sont tués avaient cherché leur mort. Par exemple, un dénommé Liberté est tué parce qu'il refuse d'épouser une Iroquoise. Il ne fallait quand même pas insulter ses «hôtes» à ce point. D'ailleurs, les Blancs capturés par les tribus du nord-est de l'Amérique recevaient normalement un bien meilleur traitement que les ennemis traditionnels. James Axtell, «The White Indians of Colonial America», William and Mary Quarterly, 32 (1975): 55-88.

68 Onze Iroquois furent tués en 1659 par des Français. Marie de l'Incarnation, op. cit., II: 140. Â part ceux qui étaient en prison, au moins six Agniers furent brûlés à Québec en 1660, RJ, XLVI: 84-100. Les Iroquois avaient aussi quatorze guerriers tués et dix-neuf blessés au Long-Sault à venger.

69 Marie de l'Incarnation, op. cit., II: 140; c'est nous qui soulignons.

70 RJ, XLI: 44-48; JJ: 192.

71 RJ, XLI: 54-64.

72 Les Relations donnent 71 victimes. RJ, XLII: 32, XLIII: 116. Marie de l'Incarnation dit qu'il y eut 6 tués et 85 capturés. Marie de l'Incarnation, op. cit., II: 108-109. Sur cet événement et la suite, on peut consulter Trigger, op. cit., II: 806-815. Il intitule cette section: "The rape of the Quebec Huron". Son affirmation que l'incapacité des Français à protéger les Hurons incita les Agniers à attaquer la Nouvelle-France n'est pas très bien étayée. 
çais de l'île ne furent pas inquiétés, les Agniers «disant qu'ils avaient la paix» avec eux ${ }^{73}$. En 1657, les Agniers et les Onontagués retournèrent pour forcer les autres Hurons à respecter la promesse faite en 1654 de les accompagner dans leur pays. Le 2 juin, quatorze familles Attignawantan s'embarquèrent avec les Agniers ${ }^{74}$. Le 16 juin, les Ahrendarrhonons se joignirent aux Onontagués ${ }^{75}$. Les hommes de ce groupe furent abattus avant leur arrivée à destination, sans que le père Ragueneau puisse les protéger ${ }^{76}$. Il ne restait à Québec qu'une tribu, vraisemblablement les Attingueenongnahacs, qui avaient refusé de partir ${ }^{77}$. Donc, en 1658 , il ne restait qu'environ 150 à 200 Hurons dans la vallée laurentienne, et ceux-ci s'étaient réfugiés à Québec pour plus de sécurité.

Voilà où en sont rendus les Hurons à la veille de la bataille du Long-Sault. Selon le Journal des Jésuites, la troupe d'Annaotaha était formée des «40 Hurons qui restaient ${ }^{78}$ Cette remarque écrit sur le coup et sans arrière-pensée est tout à fait vraisemblable. Ainsi tout ce qui restait d'hommes valides se trouvait au LongSault, et c'est cette présence qui explique l'acharnement des Iroquois à vouloir défaire complètement la force alliée. Dès la première journée du combat, les Onontagués savaient que le petit fort était occupé par des Hurons ${ }^{79}$. L'on se rappellera que l'armée s'était donné rendez-vous dans le but "d'enlever nos nouveaux chrétiens ${ }^{80}$, et voilà que les Hurons étaient aux prises avec une partie de cette armée et loin de la protection des Français. Les Iroquois ne poussaient pas leurs guerriers à prendre des risques inutiles et, devant une défense aussi coriace, ils auraient vite abandonné la partie si la victoire recherchée n'avait pas été extrêmement importante à leurs yeux. L'armée principale était déjà rendue près du lac Saint-Pierre. Seule la présence des Hurons explique qu'elle ait rebroussé chemin. Si son but était d'attaquer et de tuer des Français, ce n'est pas le groupe du Long-Sault qui l'en empêchait. Elle était rendue au coeur de la colonie, et il eût été facile et beaucoup moins risqué de fondre sur les habitations écartées et de ravager le pays. Si l'armée a remonté l'Outaouais, c'est que son objectif était de prendre Annaotaha et ses compagnons; la troupe de Dollard était complètement en dehors des préoccupations des Iroquois. Depuis sept ans, les Iroquois tentaient par tous les moyens

73 RJ, XLIII: 116.

74 RJ, XLIII: 186-196; JJ: 215.

75 RJ, XLIII: 198-206.

76 RJ, XLIV: 186.

77 RJ, XLIII: 190.

78 JJ: 284; c'est nous qui soulignons. La plupart des auteurs citent la Relation qui les décrit comme étant «l'élite de tout ce qui nous restait ici de considérable». RJ, XLV: 245.

79 Marie de l'Incarnation, op. cit., II: 155.

80 Ibid., II: 140. 
de détruire toute la colonie huronne, et voilà que c'était possible. Marie de l'Incarnation s'émerveille de ce que l'armée ennemie se soit contentée de «si peu de monde» ${ }^{81}$, mais c'est que ce petit groupe était le gibier tant recherché. Pour les Iroquois, la signification première du Long-Sault n'est pas la défaite des dix-sept Français, mais l'anéantissement de ce qui restait de guerriers hurons. Pour eux c'était une grande victoire.

Il ne restait à Québec que les femmes et les enfants, et des tentatives pour les enlever commencèrent aussitôt. À l'automne, un Huron échappé rapporte qu'il devait "conduire de nuit jusque dans le sein du Québec une trentaine d'Agnieronnons, pour [...] enlever le reste de la colonie huronne ${ }^{82}$. Cette armée qui devait venir fut découragée par un accident de chasse; le chef fut tué lorsqu'un de ses confrères tira sur un cerf ${ }^{83}$. C'était de mauvais augure, et il fallait rebrousser chemin. Ce fait démontre, encore une fois, la persistance des croyances traditionnelles qui motivaient les actions des Amérindiens.

C'est donc cet acharnement sans bornes qui fournit la meilleure explication des événements entre 1653 et 1660 . Ce comportement ne peut découler d'une motivation purement économique. Il révèle plutôt la permanence parmi les sociétés amérindiennes des conceptions traditionnelles de l'honneur, de la guerre et de la vengeance. Sans doute, des attitudes nouvelles entraînées par des nécessités économiques commencent à influencer les mentalités; mais, pendant plusieurs années, ces attitudes doivent coexister avec des valeurs traditionnelles résistantes et tenaces. Une longue tradition historiographique a refusé de tenir compte des spécificités du comportement indigène. Trop préoccupée par la création de héros pour l'édification de la jeunesse, ou par la transformation de personnages du XVIIle siècle en hommes modernes ${ }^{84}$, elle a manipulé les événements pour servir des buts immédiats sans se soucier le moindrement de la réalité amérindienne. Les nations du nordest de l'Amérique ont joué un rôle de premier plan dans l'histoire de la colonisation européenne, et elles méritent un meilleur sort. Un effort de compréhension de leur comportement jette une nouvelle perspective sur les événements et les personnages de l'«âge héroïque». Cet effort s'avère nécessaire, si l'on veut comprendre l'action entreprise par les Français pendant cette période critique de la formation d'une société distincte dans la vallée laurentienne.

81 Ibid., II: 163.

82 RJ, XLVI: 120

83 Marie de l'Incarnation, op. cit., II: 189.

84 Sur l'utilisation de l'affaire Dollard à des fins idéologiques, voir Jacques Chevalier, «Myth and Ideology in "Traditional» French Canada: Dollard, The Martyred Warrior", Anthropologica, XXI (1979): 143-175. 